\title{
Cosmic ray ensembles from ultra-high energy photons propagating in the galactic and intergalactic space
}

\author{
Niraj Dhital \\ Institute of Nuclear Physics Polish Academy of Sciences, Radzikowskiego 152, Cracow, Poland \\ E-mail: niraj.dhitaleifj.edu.pl
}

\section{Oleksandr Sushchov}

Institute of Nuclear Physics Polish Academy of Sciences, Radzikowskiego 152, Cracow, Poland E-mail: oleksandr.sushchoveifj.edu.pl

\section{Jan Pękala*}

Institute of Nuclear Physics Polish Academy of Sciences, Radzikowskiego 152, Cracow, Poland E-mail: jan.pekala@ifj.edu.pl

\section{Kevin Almeida Cheminant}

Institute of Nuclear Physics Polish Academy of Sciences, Radzikowskiego 152, Cracow, Poland E-mail: kevin.almeida-cheminanteifj.edu.pl

\section{Dariusz Góra}

Institute of Nuclear Physics Polish Academy of Sciences, Radzikowskiego 152, Cracow, Poland E-mail: dariusz.gora@ifj.edu.pl

\section{Piotr Homola}

Institute of Nuclear Physics Polish Academy of Sciences, Radzikowskiego 152, Cracow, Poland E-mail: piotr.homola@ifj.edu.pl

\section{for the CREDO Collaboration ${ }^{\dagger}$}

\begin{abstract}
Propagation of ultra-high energy photons in the galactic and intergalactic space gives rise to cascades comprising thousands of photons. Using Monte Carlo simulations, we investigate the development of such cascades in the solar magnetosphere, and find that the photons in the cascades are distributed over hundreds of kilometers as they arrive at the top of the Earth's atmosphere. We also perform similar study for cascades starting as far as $10 \mathrm{Mpc}$ away from us using relevant magnetic field models. A few photons correlated in time are expected to arrive at the Earth from the latter type of cascade. We present our simulation results and discuss the prospects for detection of these cascades with the Cosmic-Ray Extremely Distributed Observatory.
\end{abstract}

36th International Cosmic Ray Conference -ICRC2019-

July 24th - August 1st, 2019

Madison, WI, U.S.A.

\footnotetext{
*Speaker.

${ }^{\dagger}$ Full author list: http://credo.science/publications
} 


\section{Introduction}

Although a definitive conclusion on the exact composition of ultra-high energy cosmic rays (UHECR) is lacking, the current observations and the conclusions drawn thereof strongly suggest that UHECRs are hadronic in nature [1,2,3]. Analyses based on different observables measured from the extensive air showers (EASs) produced by the UHECRs suggest that the primaries appear to be more like atomic nuclei heavier than protons [2]. In addition, it has also been established that some crucial features, e.g., the muon content in EASs produced by UHECRs, do not quite agree with what is obtained from the simulations of hadron-initiated showers. More muons are present in EASs than the simulations suggest [4]. We also lack a definitive understanding of the mechanism that produces (accelerates) the primary particles at (to) such high energies. Of the two broad classes of models for UHECR production or acceleration, the bottom-up models are based on assumption that the low energy particles are accelerated to the ultra-high energies (UHE) while the top-down models stem from assumption that the UHECRs are produced as a consequence of decay or annihilation of super-massive dark matter particles or topological defects[5]. If the former is solely responsible for UHECR production we should a see very small fraction of UHE photons, while if the latter is a dominant cause of UHECR production we expect a much larger photon fraction.

The most up-to-date results from the photon searches performed by various experiments show the non-observation of UHE photons and have placed stringent upper limits [6, 7] disfavoring the top-down model of UHECR production. However, there are still compelling reasons for the UHE photon search. The conclusions from the searches performed so far are based on the interaction models which are derived by extrapolating the experimental data measured at lower energies. Eventually, the physics uncertainties grow significantly in the UHE regime, thus making it harder to correctly characterize the development of EASs. The mismatch between the muon number from observations and that from simulation results for hadronic showers also appeals for a further investigation for UHE photon scenarios. Both observation and non-observation of UHE photons will have a number of significant implications in fundamental science. It will help to establish or constrain better several aspects of contemporary astrophysics e.g., models of dark matter, Lorentz invariance violation, space-time structures etc.

We present a method of UHE photon search that is based on electromagnetic cascading of UHE photons as they propagate through space. Such cascade development can occur when the primary photon traverses some region in space with magnetic field strong enough for pair-production, and subsequently synchrotron emission from the produced electron-positron pair. As a consequence, a number of correlated particles or cosmic ray ensemble (CRE) from a UHE primary photon arrive at the Earth. The number of particles and distribution of particles in CREs depend on the distance from the Earth to the region in space where cascading takes place, as well as the strength of the magnetic field in the region.

\section{Simulations}

Using Monte Carlo simulations, we study UHE photon cascading for two different scenarioscascade development which occurs close to the Sun and that which occurs at much farther distance 
from the Earth. For the simulations of the first scenario, we use PRESHOWER 3.0 [8], while for the second scenario, we use CRPropa 3 [9].

\subsection{PRESHOWER 3.0}

PRESHOWER 3.0 is the most recent version of simulation package PRESHOWER[10], which was originally developed for the simulation of electromagnetic cascading of UHE photons in the geomagnetic field. Treatment of most of the physics processes relevant for the cascading of UHE photon in PRESHOWER 3.0 has been adopted from [10]. Some important features like a more accurate particle tracking for 3D simulation and tracking of time is added in the new version of the code. This allows one to study spatial as well as temporal distribution of photons which arrive at the top of the atmosphere. Also, PRESHOWER 3.0 allows one to study the preshowering of UHE photons not only in the geomagnetic field, but also in the other regions in nearby space (e.g., close to the Sun).

A UHE photon subject to a magnetic field $H$ produces an $e^{+} e^{-}$pair after traveling a path length $\mathrm{d} l$ with a probability,

$$
p_{\text {conv }}=1-\exp (-\alpha(\chi) \mathrm{d} l) \simeq \alpha(\chi) \mathrm{d} l
$$

where $\alpha$ is a function of variable $\chi \equiv \frac{1}{2} \frac{h v}{m_{\mathrm{e}} c^{2}} \frac{H}{H_{\mathrm{cr}}}$ with $H_{\mathrm{cr}}=4.414 \times 10^{13} \mathrm{G}$ which can be approximated by,

$$
\alpha(\chi) \approx \frac{0.16}{2 \chi} \frac{\alpha_{\mathrm{em}}}{\lambda_{\mathrm{c}}} \frac{H}{H_{\mathrm{cr}}} K_{1 / 3}^{2}\left(\frac{2}{3 \chi}\right)
$$

where $\alpha_{\mathrm{em}}$ is the fine structure constant, $\lambda_{\mathrm{c}}$ is the Compton wavelength of an electron and $K_{1 / 3}$ is a modified Bessel function. Tracking of $e^{+} / e^{-}$is performed using a modified Euler's method.

Also, the spectral distribution of energy of synchrotron photons emitted by the electrons is taken from [11],

$$
f(y)=\frac{9 \sqrt{3}}{8 \pi} \frac{y}{(1+\xi y)^{3}}\left\{\int_{y}^{\infty} K_{\frac{5}{3}}(z) \mathrm{d} z+\frac{(\xi y)^{2}}{1+\xi y} K_{\frac{2}{3}}(y)\right\},
$$

where $y=\frac{h v}{\xi(E-h v)}$ is the function of emitted photon energy $h v, \xi=\frac{3}{2} \frac{H_{\perp}}{H_{\mathrm{cr}}} \frac{E}{m_{\mathrm{e}} c^{2}}, E$ and $m_{\mathrm{e}}$ are energy and rest mass of electron respectively.

Two different models of solar magnetic field that we have used in the simulations of cascades developing close to the Sun are shown in Fig 1. For the dipole model, the magnetic field is calculated considering a dipole with magnetic moment of $6.87 \times 10^{32} \mathrm{G} \cdot \mathrm{cm}^{3}$. Although not a very realistic model, using it for the solar magnetic field in our simulations allows us to study the effects of orientation of considered dipole on the expected distribution of photons which arrive at the top of the Earth's atmosphere. The other model we used in our simulation is the so-called dipolequadrupole-current-sheet (DQCS) model [12], which gives a more realistic approximation of the solar magnetic field. 


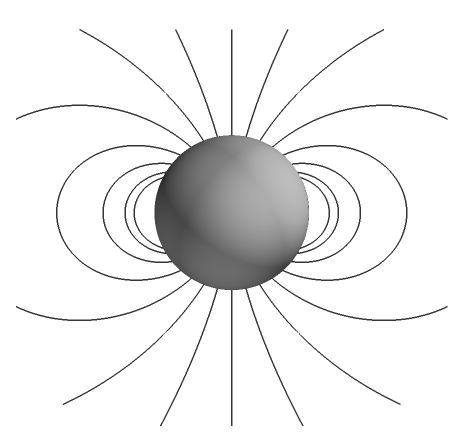

(a) Dipole field

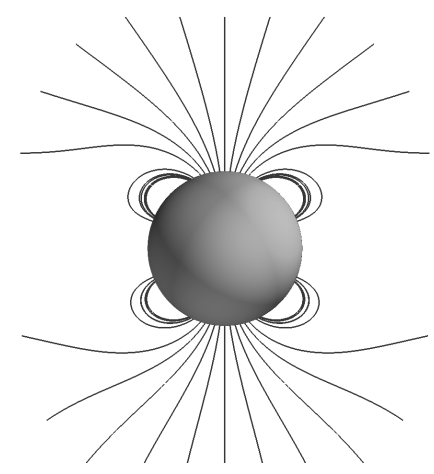

(b) DQCS field

Figure 1: Magnetic field models used in the simulation

\subsection{CRPropa 3}

CRPropa 3 is a modular simulation framework designed for studying the propagation of ultrarelativistic particles through galactic as well as extragalactic space [9]. It allows for different spatial arrangements of sources while taking into account interactions of propagating particles with background fields, their deflections in magnetic fields and cosmological effects. The modular structure provides users with a wide range of simulation options, either by combining existing modules or adding their own Python or $\mathrm{C}++$ modules.

The propagation of the injected particles (each having its starting parameters specified) is performed in discrete steps until the breaking conditions (energy threshold or reaching the observer) are fulfilled. The probability for an interaction is calculated within each propagation step with the use of the interaction length $\lambda$ of a specific process. To make the calculations computationally inexpensive, interaction rates are calculated separately for each process beforehand and implemented in the code during runtime.

\section{Results}

We summarize our results from the simulation of electromagnetic cascading of UHE photons propagating in space with regions characterized by some magnetic field. In the case of a cascade initiated close to the Sun, there are several thousand low energy synchrotron photons which are practically aligned along a line as they arrive at the top of the Earth's atmosphere. Although synchrotron emission from the electrons in the solar magnetic field (with a typical strength of around a few Gauss) is likely even at much lower energies, the pair-production of $e^{+} e^{-}$for a primary UHE photon is highly constrained by its primary energy. In Fig. 2, the magnetic pair production probability as a function of impact parameter of primary photon with respect to the Sun is shown for different energies. In Fig. 3, typical signatures of CREs produced by a UHE photon heading towards the Earth via close vicinity of the Sun are shown. The spatial extent of the photons in these CREs is several hundred kilometers. The signatures look different for different models of solar magnetic field, however, the line-signature is preserved in either of the models used. Left panel in Fig. 4 displays the spatial energy distribution of photons for the CRE in the left panel of Fig. 3. 


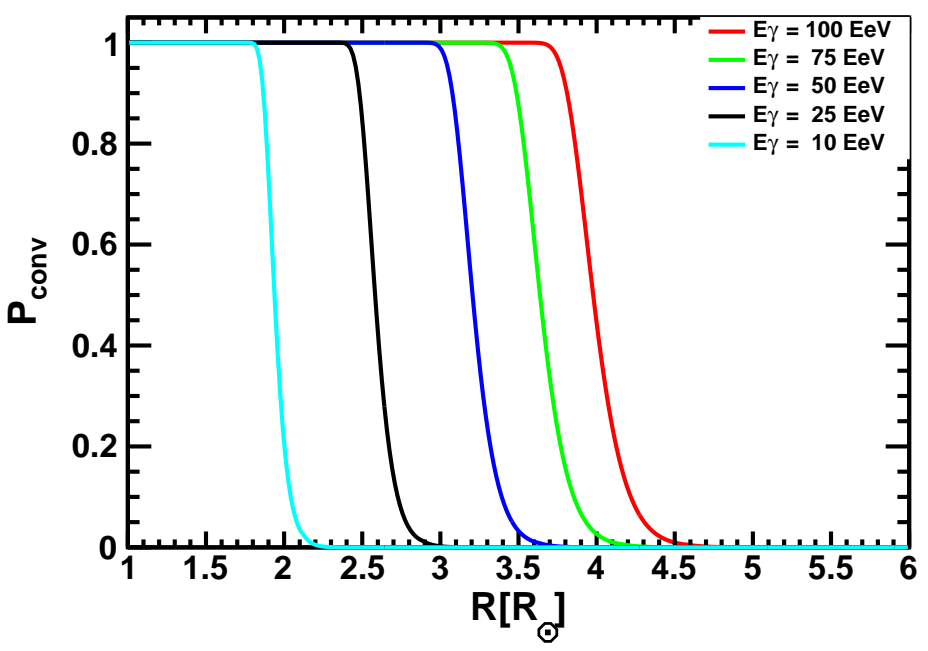

Figure 2: Probability of magnetic pair production $\left(\gamma \rightarrow e^{+} e^{-}\right)$as a function of the impact parameter for UHE photons heading towards the Earth from the Sun's vicinity.
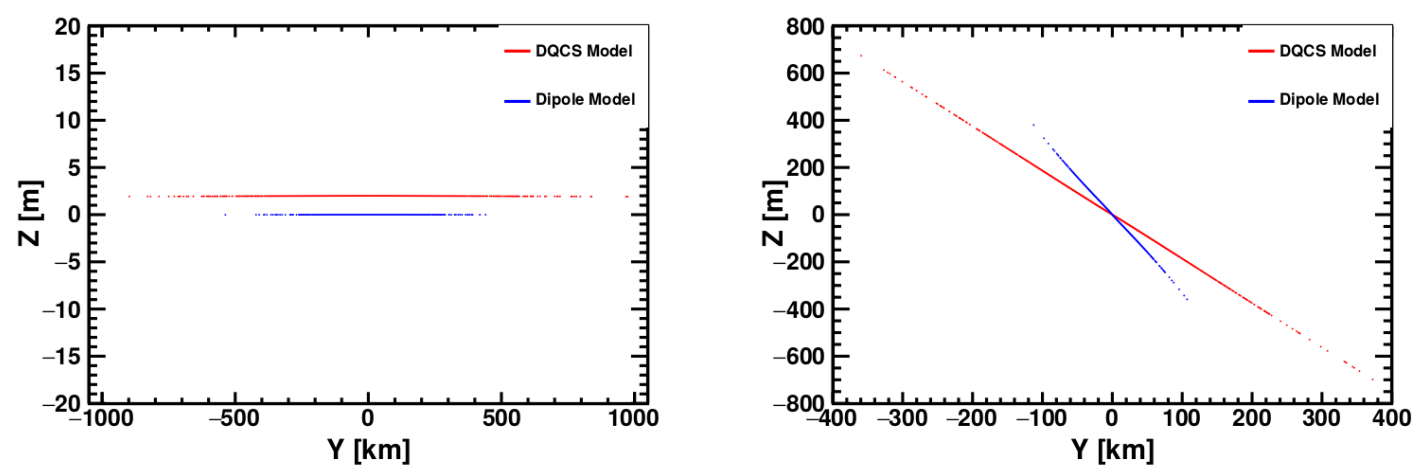

Figure 3: Spatial distribution of photons with energies $>10^{12} \mathrm{eV}$ arriving at the top of the atmosphere for a CRE produced by $100 \mathrm{EeV}$ photon. The primary photon is directed towards the Earth such that the position of the closest approach has heliocentric latitude $90^{\circ}$ (left panel) and $45^{\circ}$ (right panel). In the left panel, the distribution shown in red is shifted by $2 \mathrm{~km}$ in the positive $z$ direction.

The most energetic photons are present near the core of the CRE. On the right panel of the figure, energy distribution of the photons in the same CRE is shown.

In case of a CRE originating at a large distance from the Earth we skip the first $e^{+} e^{-}$pair production and simulate $\mathrm{EeV}$ electrons entering the galaxy, considering Jansson \& Farrar galactic magnetic field model [13]. We study the distribution of resulting photons on the observational sphere with its center at the electron's injection point, containing the Solar system on its surface. We then calculate the number of photons, reaching the surface of an Earth-sized sphere in the Solar system, thus simulating the arrival of CREs at the Earth. In the left panel of Fig. 5, an example distribution of particles on the test sphere of the Earth size is shown. In this particular example, as many as 16 photons make their way to the Earth. In the right panel, distribution of number of 

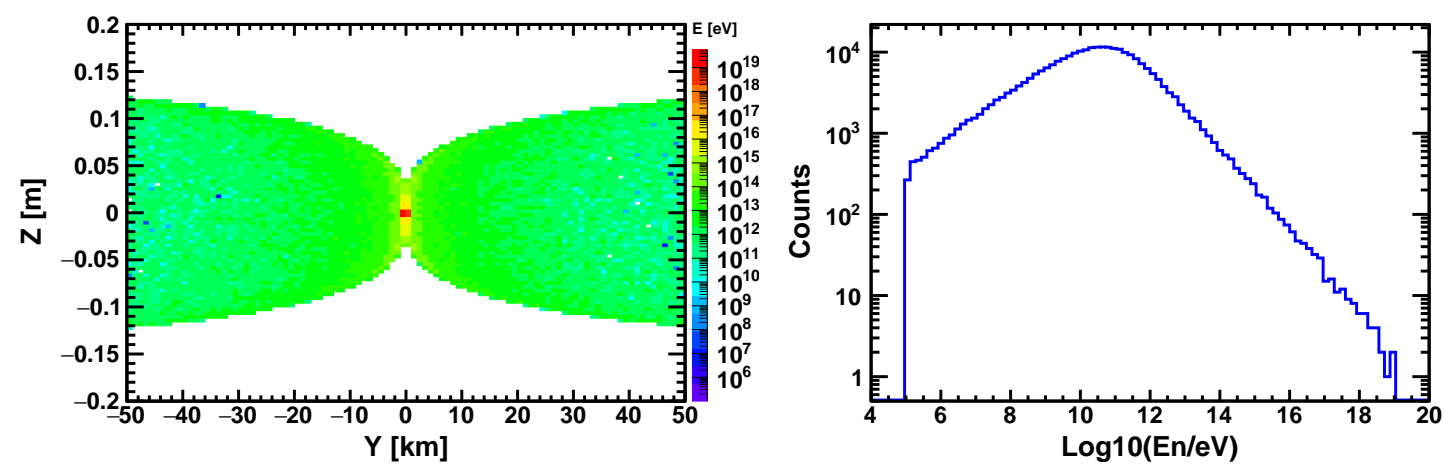

Figure 4: Distribution of energy of CRE photons arriving at the top of the atmosphere for a CRE produced by $100 \mathrm{EeV}$ photon. The primary photon is directed towards the Earth such that the position of the closest approach has heliocentric latitude $0^{\circ}$, and its impact parameter is $3 R_{\odot}$. Note the difference in the scales along $x$ and $y$ axes. Energy distribution of CRE photons with energies larger than $10^{5} \mathrm{eV}$ for the same CRE.
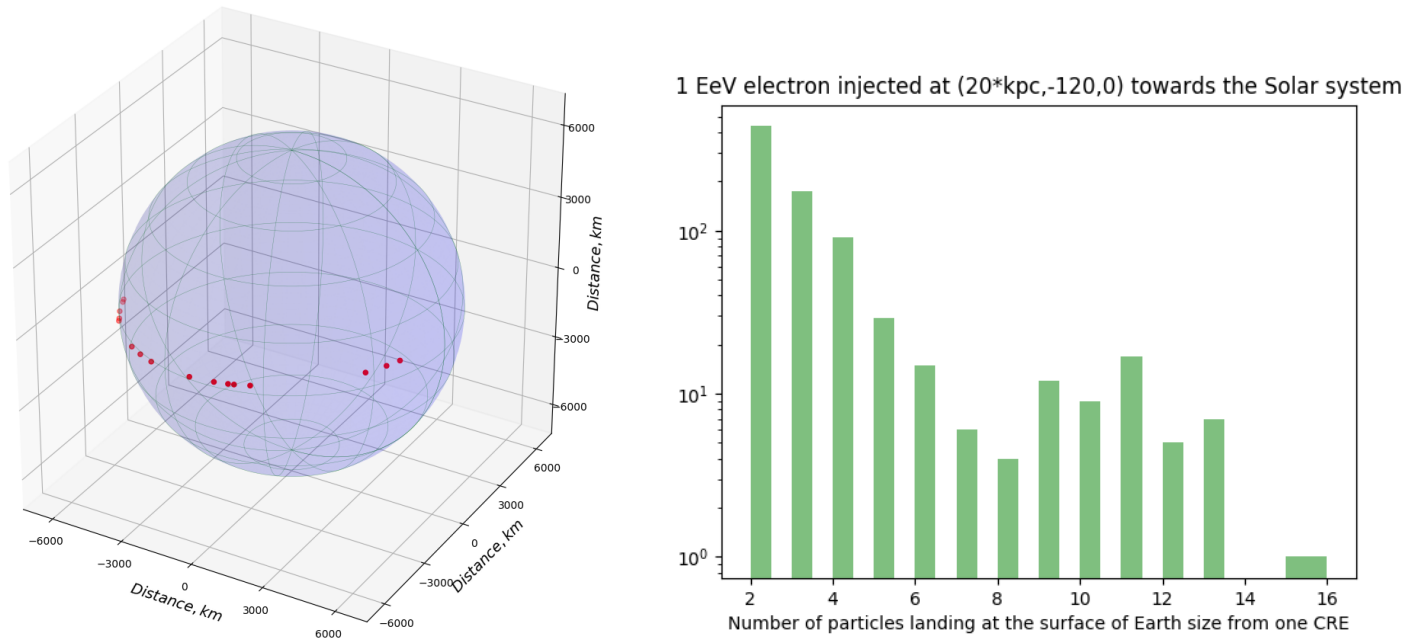

Figure 5: Left panel: Positions of CRE photons arriving at the Earth. Right panel: Distribution of number of photons with energies $>1 \mathrm{TeV}$ landing at the Earth. CRE in both cases is produced by a $1 \mathrm{EeV}$ electron entering the galaxy and heading towards the Solar system.

photons belonging to the same CRE, capable to reach the Earth "together" is shown.

\section{Summary}

If UHE photons propagate towards the Earth from their sources, they are subjected to several processes. We demonstrated through simulations that they cascade on their way while traversing regions in space with sufficient magnetic field. Instead of a single primary UHE photon, there is a possibility that a cascade of several correlated particles from a photon primary arrives at the Earth. Results from simulations performed for UHE photons traveling through the Sun's vicinity show that a large number of photons $\left(\sim 10^{4}\right)$ are produced. Photons in these CREs are spatially extended over a distance of several hundred kilometers as the CRE arrives at the top of the Earth's 
atmosphere and are distributed practically along a line. Also, they span a wide range of energy from $\mathrm{GeV}$ to $\mathrm{EeV}$.

CREs which originate at large distances can span the whole Earth, and as in the other scenario we studied, its signature is also a very extended line. However, a straight-forward detection of such signature is limited by relatively lower number of particles reaching the Earth.

CREs provide us with a unique opportunity to explore UHE photons. Contrary to the currently used common approaches which are based on observables measured in EASs produced by primary UHECRs, an alternative approach for photon search based on CREs can be used. The new approach will enable us to detect UHE photons which preshower before arriving at the Earth's atmosphere, which is difficult to do by implementing currently used common methods.

The main objective of Cosmic-Ray Extremely Distributed Observatory (CREDO) [14] is to study cosmic rays with a particular emphasis on CREs. CREs produced by UHE photons at regions close to the Sun give rise to a line-like footprint several hundred kilometers long at the top of the atmosphere, and consequently similar footprint of secondary particles is expected at the ground. For CREs produced at very far distances, we expect a few tens of correlated particles that arrive at the Earth. CREDO, which is a global network of cosmic ray detectors, will enable us to detect CREs produced in either of the scenarios.

\section{Acknowledgments}

This research has been supported in part by PLGrid Infrastructure. We warmly thank the staff at ACC Cyfronet AGH-UST, for their always helpful supercomputing support. CREDO mobile application was developed in Cracow University of Technology.

\section{References}

[1] R. U. Abbasi et al. [Telescope Array Collaboration], Mass composition of ultrahigh-energy cosmic rays with the Telescope Array Surface Detector data, Phys. Rev. D 99, no. 2, 022002 (2019).

[2] A. Aab et al. [Pierre Auger Collaboration], Depth of maximum of air-shower profiles at the Pierre Auger Observatory. II. Composition implications, Phys. Rev. D 90, no. 12, 122006 (2014).

[3] R. U. Abbasi et al. [HiRes Collaboration], Indications of Proton-Dominated Cosmic Ray Composition above 1.6 EeV, Phys. Rev. Lett. 104, 161101 (2010).

[4] A. Aab et al. [Pierre Auger Collaboration], Testing Hadronic Interactions at Ultrahigh Energies with Air Showers Measured by the Pierre Auger Observatory, Phys. Rev. Lett. 117, no. 19, 192001 (2016).

[5] M. Kachelriess, The rise and fall of top-down models as main UHECR sources, arXiv:0810.3017 [astro-ph].

[6] T. Abu-Zayyad et al. [Telescope Array Collaboration], Upper limit on the flux of photons with energies above $10^{19} \mathrm{eV}$ using the Telescope Array surface detector, Phys. Rev. D 88, no. 11, 112005 (2013).

[7] A. Aab et al. [Pierre Auger Collaboration], Search for photons with energies above $10^{18} \mathrm{eV}$ using the hybrid detector of the Pierre Auger Observatory, JCAP 1704, no. 04, 009 (2017).

[8] N. Dhital et al., Simulation of ultra-high energy photon propagation with PRESHOWER 3.0, (in preparation). 
[9] R. Alves Batista et al., CRPropa 3 - a Public Astrophysical Simulation Framework for Propagating Extraterrestrial Ultra-High Energy Particles, JCAP 1605, no. 05, 038 (2016).

[10] P. Homola et al., Simulation of ultrahigh energy photon propagation in the geomagnetic field, Comput. Phys. Commun. 173, 71 (2005).

[11] A. A. Sokolov, I. M. Ternov and C. W. Kilmister, Radiation From Relativistic Electrons, NEW YORK, USA: AIP (1986) 312 P. (AIP TRANSLATION SERIES).

[12] M. Banaszkiewicz, W. I. Axford and J. F. McKenzie, An analytic solar magnetic field model, A \& A 337 (1998) 940.

[13] R. Jansson and G. R. Farrar, A New Model of the Galactic Magnetic Field, Astrophys. J. 757, 14 (2012) doi:10.1088/0004-637X/757/1/14 [arXiv:1204.3662 [astro-ph.GA]].

[14] D. Góra for the CREDO Collaboration, Cosmic Ray Extremely Distributed Observatory: Status and perspectives of a global cosmic ray detection framework, these proceedings. 